Investigaciones Fenomenológicas, n. 13, 2016, 65-87.

e-ISSN: $1885-1088$

\title{
CONTRIBUCiones de LA FENOMENOLOGÍA DE Edith STEIN AL PROBLEMA MENTE-CUERPO: APUNTES PARA LA PSICOLOGÍA
}

\author{
Contributions of Edith Stein's PHENomenology to \\ THE MIND-BODY PROBLEM: NOTES FOR PSYCHOLOGY
}

\begin{abstract}
Resumen: La psicología es una rama científica relativamente nueva y a la que todavía le faltan bases metodológicas consistentes para fundamentar sus investigaciones. Dada su inmadurez, tal ciencia tiene dificultades para delimitar su estatuto ontológico, lo que genera diversos equívocos epistemológicos y metodológicos. Así, se impone una cuestión fundamental para la aclaración del objeto de la psicología: el problema mente-cuerpo. En ese sentido, el objetivo de este artículo, basado en la fenomenología de Edith Stein, es analizar algunos trabajos de la filósofa, a saber: "Causalidad Psíquica" e "Introducción a la Filosofía". De esa manera, a través de las investigaciones de Stein acerca de la psique (que puede situarse en la cercanía de lo que se entiende por mente en el ámbito de las ciencias de la mente) y del cuerpo, se concluye que es posible concebir la cuestión psique/mente-cuerpo como una unidad-dual que se instancia en el Leib.
\end{abstract}

Palabras clave: Problema mente-cuerpo, fenomenología, Edith Stein

\author{
Tommy Akira Goto \\ Universidade Federal de Uberlândia \\ Uberlândia (Minas Gerais, Brasil) \\ Mak Alisson Borges de Moraes \\ Universidade Federal do Triangulo Mineiro \\ Uberaba (Minas Gerais, Brasil) \\ prof-tommy@hotmail.com \\ makalisson@hotmail.com
}

\begin{abstract}
Psychology is a relatively new scientific branch that still lacks consistent methodological foundations to support their investigations. Given their immaturity, that science still faces difficulties to delimit its ontological status, which raises various epistemological and methodological misconceptions. Thus, there is a fundamental question for an elucidation of the object of Psychology: the mindbody problem. The proposal of this article, in this sense, is to discuss the mind-body problem in the light of the Phenomenology of Edith Stein, seeking from there lay the fundation of Psychology. For this, some works of the philosopher was analysed, namely: "Psychic Causality" and "Introduction to Philosophy". In this way, through the investigations of Stein about the psyche (which can be approximated to what is meant by mind in the context of the Sciences of the mind) and body, concluded that it is possible to conceive the psyche/mind-body issue while a dual-unit that instantiates in the Leib.
\end{abstract}

Keywords: Mind-body problem, phenomenology, Edith Stein 


\section{INTRODUCCIÓN}

Cuando se ponen de manifiesto las fragilidades epistemológicas y ontológicas de la psicología científica expuestas por Edmund Husserl (1859-1938), se constata que la fenomenología se caracteriza como la base metodológica que provee un fundamento seguro para esa ciencia. La metodología fenomenológica pone las bases para un esclarecimiento riguroso del objeto de estudio de la psicología, diseñando, de esa manera, su estatuto ontológico. Husserl propuso una refundación de la psicología por medio de su proyecto de una psicología fenomenológica, posibilitando la constitución de una especialidad auténticamente científica.

Al proponerse como el fundamento metodológico de la psicología, la fenomenología se dispone a su vez a afrontar el problema mente-cuerpo. En el actual contexto de las ciencias cognitivas, la fenomenología ha sido requerida para contribuir a pensar sobre esa cuestión, cuya dificultad ha propiciado la evolución hacia el proyecto de naturalización de la fenomenología, que presenta equívocos que acaban poniendo ese programa en jaque, como lo han destacado Bello (2012, p. 14), Depraz (1999, p. 471), Goto (2015, p. 140), Simanke (2012, p. 328), Sidoncha (2008, 371) y Zahavi (2010, p. 04). Es por ello necesario cuestionarse la posibilidad de una reflexión desde la fenomenología acerca del problema mente-cuerpo que no desemboque en una naturalización del proyecto fenomenológico que lo aborda.

La fenomenología de Edith Stein (1891-1942) puede ser incluida en el ámbito de las discusiones del problema mente-cuerpo. En este trabajo intentaremos abordar esa problemática a partir de las reflexiones de Stein sobre la psicología y sus investigaciones sobre la persona humana. Desencantada con la psicología científica naturalista de su época, a la que llamó "psicología sin alma", Stein comprobó que a esa ciencia le faltaban bases adecuadas para llegar a sus conocimientos, por lo que se interesó en la fundamentación de la psicología, cuestión que era una de sus grandes preocupaciones, para la que utilizó la fenomenología de su maestro.

Es necesario aclarar, en primer lugar, que, aunque Stein no ha tratado de modo directo en sus escritos la cuestión mente-cuerpo, no por ello se ha de concluir que las ideas esbozadas por su fenomenología no puedan auxiliar a pensar ese dilema; al contrario, no solo es posible, sino necesario contribuir con 
el rico pensamiento de Stein a esas discusiones actuales. Teniéndolo en cuenta, y antes de empezar la discusión, son indispensables algunas clarificaciones de los términos que aparecen en su análisis. Se señala que la filósofa no empleó el término mente ( $m i n d$ ) en el sentido en que ha sido aplicado en las discusiones actuales acerca del problema mente-cuerpo en el ámbito de la Filosofía de la Mente y de las ciencias cognitivas. Al referirse a la mente, utilizó la palabra latina Mens/Spiritus, la cual nombra, siguiendo la tradición aristotélico-tomista, la parte superior del alma (Stein, 2002a, p. 120).

En virtud de ello, para resaltar las posibles contribuciones de la fenomenología de Stein al problema mente-cuerpo, se realizará aquí un acercamiento terminológico entre los términos mente, tal y como es comprendido en el área de las ciencias de la mente, y psique, que, en el pensamiento de la filósofa, alude a la dimensión del ser humano relativa a los fenómenos psicológicos. Se entiende que los dos términos indican el objeto de estudio de la psicología. Así, aunque existan diferencias entre la investigación fenomenológica y las investigaciones en el ámbito de las ciencias de la mente, se subraya que ambas quieren esclarecer el objeto psicológico (Mangarano, 2012, p. 329).

Además, es indispensable destacar también una cuestión lingüística que justifica ese acercamiento. En la lengua alemana no se encuentra un vocablo correspondiente directo para el término 'mente'. Al referirse al objeto de la psicología, los pensadores alemanes suelen utilizar la palabra Seele (alma) o Psyche (psíque). Por consiguiente, además de las diferencias epistemológicas, la fenomenología y las ciencias de la mente asimismo presentan disparidades lingüísticas, las cuales, empero, no impiden una interlocución entre ellas. Teniendo en cuenta esa circunstancia, es legítimo hacer una aproximación de léxico entre los vocablos mente y psique (Araujo, 2007, p. 95). Se puede suponer, entonces, que el dilema mente-cuerpo en el ámbito de la fenomenología de Stein puede manifestarse como un problema psique-cuerpo.

Por consiguiente, para comprender la cuestión psique/mente-cuerpo basándonos en el pensamiento de Stein, recurriremos a la antropología fenomenológica trazada por la filósofa, exponiendo sus investigaciones acerca de la psique, del cuerpo y de cómo se puede engendrar el vínculo entre esas dos estructuras. Con el fin de mantener la discusión, serán analizadas aquellas obras en que la fenomenóloga inspira una investigación fecunda sobre el ser humano a través del método fenomenológico, contribuyendo de manera significativa a 
una fundamentación de la psicología: "Causalidad psíquica" (Psychische Kausalität), presente en su obra Contribuciones a una fundamentación filosófica de la Psicología y las Ciencias del espíritu (Beiträge zur philosophischen Begründung der Psychologie und der Geisteswissenschaften, 1922); Introducción a la filosofia (Einführung in die Philosophie, 1920) y La estrutura de la persona humana (Der Aufbau der menschlichen person, 1932).

\section{LA DIMENSIÓN PSÍQUICA}

Al comienzo de su vida académica, Stein se interesó por la psicología, asistiendo a las clases del psicólogo William Stern (1871-1938). Sin embargo, inmediatamente vio las limitaciones de esa ciencia, que, a causa de su impostación naturalista, no lograba investigar adecuadamente la esencia de la psique, necesitando todavía de bases seguras para obtener sus conocimientos. Tras sus estudios de Psicología en Breslau, la filósofa concluyó que: "Todos mis estudios de psicología me habían llevado al convencimiento de que esta ciencia estaba todavía en pañales; que le faltaba el necesario fundamento de ideas básicas claras, y que esta misma ciencia era incapaz de elaborar esos presupuestos" (Stein, 2002b. p. 331).

Debido a su impronta naturalista, la psicología excluyó las cuestiones concernientes al mundo anímico, reduciendo lo psíquico a algo solamente material y entendiéndolo como un simple conjunto de sensaciones sin ningún sentido para la vida humana. De acuerdo con Stein (2007, p. 1130), la psicología se apartó del flujo de la vida anímica, renunciando a su sentido originario de investigar el "mundo interior", lo que la condujo a una crisis que la mostraba desprovista de apoyos seguros.

En consecuencia, para reconocerse como una verdadera ciencia, la psicología necesitaba primeramente clarificar qué es su objeto de estudio, es decir, lo psíquico. Se puede percibir que a lo largo de la historia de la psicología moderna la cuestión de la elucidación de su objeto no ha sido tratada de forma sistemática y rigurosa, lo que llevó a diversos equívocos acerca de la cuestión. Y en consecuencia, surgieron distintas psicologías, lo que dio lugar a una variedad desordenada de concepciones sobre lo psíquico. Era relevante volver a iniciar una investigación auténtica acerca de la dimensión psíquica, buscando una 
aclaración adecuada del objeto de estudio de la psicología, lo que sobrepasaba los equívocos metodológico-epistemológicos de esa ciencia.

Es suma, había que constatar que el método fenomenológico se erigía en una herramienta apropiada para esa investigación de lo psíquico. De ahí que, siguiendo los análisis de Husserl en cuanto a la psicología, Stein delinease una psicología fenomenológica con el objeto de clarificar qué es lo psíquico o, de manera más precisa, cómo el individuo psíquico se presenta. De ese modo, se estaría en condiciones de establecer una psicología auténticamente científica y de subrayar su lugar en relación con las demás ciencias (naturales y del espíritu).

\subsection{Psique y Conciencia}

La investigación de Stein (2005a, p. 217) respecto a la psique empezó desde un análisis de la llamada causalidad psíquica. Ese tema remite a la antigua disputa entre determinismo e indeterminismo, o en otros términos, entre necesidad y libertad. ¿Sería la voluntad dependiente de una legalidad causal? ¿O se tiene una ilimitada libertad de la voluntad, de manera que no sea posible concebir ninguna especie de necesidad? La filósofa afronta esas cuestiones, buscando analizar cómo la psique ingresa en el ámbito de la legalidad causal.

Edith Stein pretendió investigar si lo psíquico presenta su vínculo propio ineludible de manera análoga al vínculo causal en el ámbito de la naturaleza física. Entregándose a la investigación de esta problemática, afrontaba el problema de la relación o enlace entre lo psíquico y lo físico. En lo que respecta a esa cuestión, históricamente aparecen las soluciones clásicas del paralelismo psicofísico y la teoría de la interacción; pero ambas posiciones, tanto el paralelismo como la mirada interaccionista, presentan un carácter dual.

En su investigación acerca, por un lado, de la relación entre lo psíquico y lo físico y, por otro, del vínculo causal en el ámbito psicológico, Stein comprobó la falta de claridad respecto a lo qué es lo psíquico. Aunque los manuales de psicología hayan tratado esa cuestión, lo han hecho de forma superficial, cayendo en un equívoco fundamental: la confusión entre conciencia y psique. Según la fenomenóloga, lo psíquico se refiere al "Yo - real" (empírico), o sea, el yo constituido por estados y cualidades reales, mientras que la psique debe ser entendida como una realidad trascendente. 
En este punto Stein estaría de acuerdo con la idea propuesta por Husserl (2005, p. 159) en el segundo tomo de Ideas, a saber, que lo psíquico se constituye como una realidad anímica, de manera que se tienen dos especies de experiencia real: de un lado, la de las cosas materiales en cuanto realidades materiales $y$, de otro, la experiencia anímica como experiencia de una realidad anímica. Así, Stein señala que lo psíquico (realidad anímica) es el "Yo - real" o psicológico, es decir, el individuo real dotado de una posición empírica en el mundo, conforme lo que escribió la filósofa: "Como un óv [ente] del mundo real, la psique se inserta, lo mismo que la cosa material, en las categorías supremas de la realidad" (Stein, 2005a, p. 799).

En cambio, de modo distinto a lo psíquico, se encuentra la conciencia como el dominio de las vivencias puras, libres de cualquier impostación empírica. Lo que se sabe de la conciencia pura viene del residuo fenomenológico producto de la reducción trascendental. Al buscar un soporte seguro para el conocimiento, Husserl, aplicando el método fenomenológico, redujo el "Yo-empírico" (psicológico), accediendo de esa manera a la esfera pura de lo trascendental. La conciencia trascendental no presenta cualidades empíricas, sino trascendentales, y revela al "Yo-Puro" (Reines Ich), punto de irradiación de las experiencias puras (Stein, 2005a, p. 220).

En definitiva, mientras la psique se refiere al "Yo-empírico", es decir, al individuo poseedor de cualidades reales (realidad anímica), la conciencia se refiere al "Yo-puro", a la esfera trascendental que produce las vivencias puras. A pesar de ser diferenciables, la psique y la conciencia tienen un vínculo. La conciencia, a causa de su carácter constitutivo, constituye la realidad anímica. Utilizando la metáfora de Bello (2015, p. 28), la conciencia puede equipararse con un vidrio: por medio de ese vidrio se aprehende todo lo que está fuera de él y, de esa forma, la psique, en cuanto realidad anímica, está fuera del vidrio/conciencia, se capta y constituye por medio de él.

En la diferencia establecida por Husserl y Stein entre psique y conciencia está el origen de otra importante distinción: la disparidad entre psicología y fenomenología. De acuerdo con lo que mostró la filósofa, los manuales de Psicología confunden el concepto de lo psíquico con el de conciencia, lo que causa graves errores metodológicos en el ámbito de la investigación psicológica. De ahí que, al establecer las diferencias entre psique y conciencia, sea posible aclarar de manera precisa las áreas de investigación de la psicología y de la 
fenomenología (Stein, 2005a. p. 221). Pese al hecho de que Husserl, en la primera edición de las Investigaciones Lógicas, haya identificado la fenomenología con una psicología descriptiva, posteriormente, en la segunda edición de 1913, en las Ideas y en el artículo "La filosofía como ciencia estricta", promovió una separación entre las investigaciones fenomenológica y psicológica.

Ciñéndonos a sus propuestas, la psicología tiene como objeto de estudio lo psíquico; su tarea consiste en investigar la dimensión psíquica y las leyes que la rigen. Por otro parte, la fenomenología trascendental se responsabiliza del estudio de la conciencia, puesto que pretende hacer una aclaración de la legalidad del proceso de constitución en el ámbito de la conciencia pura (Husserl, 1965, p.4; 2006, p.116). A partir de esas especificaciones, Stein comentó que:

La diferencia entre fenomenología y psicología, exigida por Husserl en las Ideen y anteriormente en el artículo Philosophie als strenge Wissenschaft, publicado en la revista "Logos", se fundamenta en la separación requerida entre la conciencia y lo psíquico. La psicología, en el sentido de este deslinde y a la vez en el sentido que le dan los psicólogos que la practican con ingenuidad, sin consideraciones epistemológicas sobre el método adoptado, es una ciencia "natural" o "dogmática", una investigación teórica de determinados objetos que hallamos en "el mundo", en nuestro mundo, en el que vivimos y cuya existencia es el primer dogma y el presupuesto más evidente no indagado de todas nuestras reflexiones. En este mundo, además de cosas naturales y organismos vivos, encontramos seres humanos y animales, que, aparte de lo que tienen en común con las cosas y con los simples seres vivos, muestran también ciertas características que los distinguen singularmente. A la totalidad de esas características la denominamos lo psíquico, y su investigación es tarea de la psicología. (Stein, 2005a, p. 221).

Una vez establecida esa distinción entre conciencia y psique, resulta ya posible ahora investigar la dimensión psíquica por sí misma, sin incurrir en confusiones conceptuales. No obstante, como se evidenció anteriormente, la psique es una realidad anímica trascendente constituida por lo que es captado por la conciencia. Dicho de otro modo, solamente es posible conocer la psique por medio de la actividad de la conciencia. La investigación fenomenológica, al tomar la conciencia constituyente como fundamento, posee las vivencias como punto de partida. 
De esa manera, la investigación de Stein con respecto a lo psíquico se inicia en la última conciencia constituyente, a saber, en la corriente de conciencia originaria o corriente de experiencias. Ese análisis presenta una gran vinculación con las lecciones husserlianas acerca del tiempo, con las que la filósofa estuvo en contacto directo. De acuerdo con lo que destacó Stein, la corriente originaria de la conciencia se establece como un puro devenir, un flujo continuo en el cual no hay una sucesión de fases de manera que la precedente cause la subsiguiente. Por lo tanto, se concluye que la corriente originaria de la conciencia no presenta un nexo causal entre las fases, ya que ocurren en un flujo en que en el momento actual se encuentra tanto lo que ya transcurrió, como lo que está porvenir.

\subsection{El mecanismo psíquico}

Al comprobar que en el dominio de la corriente originaria de la conciencia no hay vínculo causal, Stein quiso verificar si podría haber una causalidad en el ámbito de las vivencias psíquicas. En sus reflexiones, la filósofa constató que existe una conexión entre las experiencias, puesto que los cambios en la esfera de los sentimientos vitales acarrean cambios en los transcursos de aquellas.

Parece razonable, pues, que cupiese preguntar si esas alteraciones de lo vivido responden a una relación causal. ¿Posee la esfera psíquica una causalidad? En caso de que la contestación sea positiva, ¿cómo se establece? ¿Es idéntica a la causalidad física o presentaría matices propios? Para contestar a esas preguntas, Stein profundizó en su investigación de la psique, con el objeto de desvelar su mecanismo. En su opinión, se pueden comprender esas modificaciones en el ámbito de lo vivido como una causalidad en la esfera de las vivencias. De manera análoga a los nexos causales en el mundo físico, hay en el ámbito de la psique una causalidad psíquica. En el campo de la realidad física, se explican los vínculos causales de manera que un acontecimiento causal está compuesto por un suceso causante y un suceso causado, de modo que se produzca un sobrevenir que promueva el pasaje del primero al segundo. Es en ese sentido como al liberar un objeto a una determinada altura (sobrevenir causante), él será atraído en dirección al suelo (sobrevenir causado) debido a la fuerza de la gravedad (causa).

A semejanza de lo que sucede en el mundo físico, en el dominio psíquico también hay relaciones causales. Por consiguiente, como subrayó Stein (2005a, p. 246), un impulso puede generar una serie de cambios en el trascurso de las 
experiencias, lo que determina no solo la calidad, sino también la intensidad del efecto resultante. Sin embargo, la filósofa señala que, aunque haya analogía con el mundo físico, la causalidad psíquica posee aspectos propios que la diferencian de la causalidad natural. Esa cuestión es importante para la comprensión de la esencia de lo psíquico, ya que evidencia que la psique, aunque forme parte de la realidad como también lo hace el mundo material, se distingue de él al presentar un carácter eidético propio.

Acabamos de exponer uno de los argumentos que hizo a Stein (2005a, p. 220) oponerse a la Psicología de su época que, al adoptar una concepción naturalista de la dimensión psíquica, buscaba conocer los fenómenos psíquicos por su medición. Desde esa perspectiva, la psicofísica (psicología experimental), por ejemplo, investigaba el vínculo entre estímulos físicos y experiencia sensorial, utilizando la metodología de las ciencias naturales, tratando de alcanzar la exactitud mediante la medida. Ese tratamiento manifiesta la falta de claridad respecto al objeto de estudio de la psicología (lo psíquico) y los consecuentes errores metodológicos subsiguientes.

Tras la argumentación expuesta respecto a la primera cuestión sobre la existencia o no de una causalidad en la esfera de lo psíquico, es conveniente aclarar el mecanismo de esa causalidad. Las relaciones causales en el ámbito de la psique se presentan como una concatenación de experiencias; y para aprehender cómo sucede, es necesario analizar detalladamente la manera en que ese proceso se estructura.

Para Stein (2005a, p. 240), el vínculo causal entre las vivencias sobreviene desde los cambios en la esfera vital: se señala aquí la existencia de una esfera vital que influye causalmente en las vivencias. Stein apuntó el cansancio y el frescor como dos límites extremos de esa vitalidad, que, desde esos dos climas, presenta diversos grados de variaciones, recorriéndose como un continuum.

La esfera vital, fundamento de la dimensión psíquica, exhibe una amplia variedad de grados de vitalidad. Se puede ir desde un estado de fuerte excitación hasta uno de extremo letargo, con lo que tales modificaciones exhiben cambios causales en las vivencias, indicando la existencia de un mecanismo causal en la dimensión psíquica. Así, para comprender rigurosamente la causalidad psíquica, es indispensable lanzarse a una investigación de la esfera vital. ¿Qué es esa esfera y cómo funciona? ¿De qué manera influencia los nexos causales del vivenciar? 
En el entorno de la esfera vital se encuentran los estados vitales y los sentimientos vitales que, según Stein (2005a, p. 236), tienen el mismo significado. Los estados vitales hablan del modo actual en que se encuentra la esfera vital y, a su vez, los sentimientos vitales componen la vivencia de esos estados, en otras palabras, son manifestaciones en la conciencia. No obstante, es primordial resaltar la posibilidad de que se den estados vitales que no se expresan en sentimientos vitales. En resumen, que no se expongan a la conciencia y por eso no sean vivenciados; por ejemplo, puede existir un estado de cansancio sin que se sepa de su existencia.

En virtud de ello, se indica que la esfera vital expresa las cualidades de una realidad. Los distintos sentimientos vitales hablan respecto a las condiciones momentáneas de la esfera vital (estados vitales) y manifiestan, de esa manera, una cualidad real fija: la fuerza vital. Así, la realidad psíquica, eso es, la dimensión psíquica, está compuesta por una cualidad real que Stein nombró como energía vital. En las palabras de la filósofa:

En los sentimientos vitales como contenidos inmanentes se manifiestan - de manera parecida a como sucede con los datos extraños al yo- las condiciones de una realidad, sus estados y cualidades. Así como en las sensaciones de colores el color de una cosa se manifiesta como su estado óptico momentáneo, y en el cambio de tales estados se manifiesta la permanente cualidad óptica, así también en el sentimiento vital se manifiesta una condición momentánea de mi yo - su estado vital-, y en el cambio de tales condiciones se manifiesta una cualidad real permanente: la energía vital (Stein, 2005a, p. 237).

En síntesis, la esfera vital (psíquica) exhibe distintos estados y sentimientos vitales, de manera que esas variaciones desvelan la existencia de una fuerza vital. Así, al darse cuenta de que ahora se está agotado y, poco tiempo después, excitado, se confirma como sustrato de esos distintos estados la presencia de la fuerza vital. En el estadio de agotamiento, la fuerza vital se muestra reducida mientras que en el frescor está elevada. De ahí que la aclaración del mecanismo psíquico dependa de un entendimiento de la función de esa fuerza en la esfera psíquica.

Según Stein (2005a, p. 239), los distintos modus en que la fuerza vital se presenta apelan al suceso causal en la esfera psíquica. Cuando suceden, los cambios en la esfera vital se definen como las alteraciones de esa fuerza, los distintos estados vitales son consecuencia de un incremento o disminución de la 
fuerza vital. La filósofa explica el funcionamiento de la causalidad en el ámbito psíquico:

El hecho de que se proporcionen o se sustraigan energías a la energía vital es la "causa" del acontecer psíquico. El "efecto" consiste en los cambios de las demás cualidades psíquicas. No existe una dependencia causal directa entre otras cualidades entre sí, sin que exista una mediación de la energía vital. Por ejemplo, la receptividad para los colores no puede quedar intensificada ni reducida por la receptividad para los sonidos. Pero ambas pueden quedar intensificadas conjuntamente por medio de una intensificación, independiente de ambas, de la energía vital (Stein, 2005a, p. 239).

Tras esclarecer cómo ocurre la causalidad en la esfera psíquica, ahora es posible entender cómo sucede el mecanismo psíquico. Sin embargo, es necesario subrayar también el polo opuesto de esa causalidad, que no es otro que el suceso de que los estados psíquicos consumen la fuerza vital, lo que provoca, a su vez, cambios en la esfera vital. Así, si por un lado el vivenciar presenta una dependencia causal de la fuerza vital, por otro lado se señala que los estados psíquicos dependen de un consumo de esa fuerza, ocasionando modificaciones en la dimensión psíquica. Stein afirma que: "Tenemos aquí en realidad una especie de «retroacción», pero que no representa nada nuevo con respecto a otras relaciones causales. (...). Se trata de una bilateralidad que es propia de todo acontecer causal" (Stein, 2005a, p. 240).

En el estado de frescura, hay una aplicación de la fuerza vital, visto que las experiencias se encadenan con vivacidad, de manera que la lectura de un libro, por ejemplo, es capaz de volverse una actividad entusiasta y agradable. No obstante, el propio acto de leer exige un uso de la fuerza vital que posibilita un cambio de la frescura al cansancio. Aunque la lectura sea placentera, al final de la actividad se puede estar con cansancio debido al gasto de la fuerza vital utilizada. De ahí que todo el hecho causal psíquico involucra una transposición de fuerza vital al acto de vivir, al mismo tiempo en que el propio vivir promueve un consumo de ella. Así, la demanda elevada de energía de una determinada cualidad ocasiona una fragilidad de las otras. De esa manera, por ejemplo, si alguien se concentra demasiado en algo, esa actividad exige gran cantidad de fuerza vital, llevando consigo que las otras tareas sean perjudicadas.

En cambio, sí hay una situación en la que la actividad psíquica puede ocurrir "sin esfuerzo", es decir, sin dispendio de fuerza vital. Sobreviene en el mo- 
mento en que la receptividad de la esfera psíquica se aplica a determinados contenidos por medio de la ampliación en el ámbito de la vivencia o por la intensificación de esos contenidos en las experiencias. La receptividad se muestra como una cualidad de la psique, que presenta, según Stein (2005a, p. 244), el siguiente mecanismo: amplificar el vivir o intensificar los contenidos de las vivencias exige, en un primer momento, una adquisición de fuerza vital, pero, si esa amplificación e intensificación se mantienen fijas, se crea una receptividad en la esfera psíquica de manera que el vivenciar surge sin esfuerzo. En resumen, cuanto mayor sea la receptividad, menor es el consumo de fuerza dependiente (Stein, 2005a, p. 244).

Una situación que ejemplifica ese mecanismo psíquico son los procesos de aprendizaje: al aprender algo nuevo se utiliza gran cantidad de fuerza vital, ya que la receptividad para aquella actividad es mínima. Con la práctica frecuente de lo que se ha aprendido, la receptividad crece, haciendo que la acción sea hecha sin esfuerzo. Cuando se aprende a tocar algún instrumento musical, al principio está claro el gran uso de la fuerza vital, pero, cuando se adquiere la práctica, la actividad ocurre de manera automática y espontánea (Stein, 2005a, p. 244).

En definitiva, cuando una acción se ejecuta sin esfuerzo, la fuerza vital que ha sido desprendida anteriormente, se queda disponible para la elaboración de otra actividad. En caso de que no hubiera ese mecanismo, la actividad psíquica requeriría una gran cantidad de fuerza, causando intenso deterioro de la esfera vital. Se concluye que la dimensión psíquica, en cuanto esfera pasiva, se pone como un mecanismo que se regula automáticamente.

Stein (2005a, p. 245) concluye que "no hay realidad psíquica sin causalidad" y que el mecanismo causal de la esfera vital está en la base de toda la actividad psíquica. De esa manera, la constitución de la psique con sus calidades y estados reales acontece por medio de ese proceso causal psíquico. La fenomenóloga constató que no solo hay una causalidad en la dimensión psíquica, cuyo sustrato es la fuerza vital, sino que el propio mecanismo psíquico se fundamenta en ese suceso causal.

\section{LA DIMENSIÓN CORPÓREA: CUERPO FÍSICO Y CUERPO VIVIDO}

Tras la descripción fenomenológica que Stein hace de la dimensión psíquica es relevante seguir la investigación, teniendo en cuenta la transición de lo psí- 
quico a lo psicofísico. En la concpeción de Stein (2005b, p.789), la psique no existe de manera aislada, sino solamente asociada al cuerpo. Por consiguiente, para la filósofa no es posible un entendimiento adecuado de la psique si la psicología no tiene en cuenta su íntimo vínculo con la dimensión corpórea. El "Yoempírico", dotado de una posición real, solo ingresa al mundo natural por medio del cuerpo, a saber, constituyéndose como un sujeto psicofísico.

Por esta razón, y para un examen riguroso del problema psique/mentecuerpo, es preciso emprender también un análisis fenomenológico del cuerpo, permitiendo así una adecuada aclaración de su asociación con la psique. Así pues, una psicología que pretenda asentarse sobre bases sólidas no puede rechazar la dimensión del cuerpo.

Ante todo, se entiende el cuerpo en cuanto cosa material. Para captar ese matiz de la corporeidad, Stein realiza la abstracción de los aspectos vividos del cuerpo. Se hace necesario, pues, para imaginar al hombre en su peculiaridad corporal, señalar el carácter material del cuerpo. Así como los demás objetos de la realidad natural, el cuerpo es una cosa espacial dotada de extensión. Esa faceta simplemente material del cuerpo se hace patente en el cuerpo sin vida, esto es, el cadáver, que no se diferencia de las demás cosas materiales (Stein, 2002a, p. 39; 2005b, p. 788).

En el análisis fenomenológico, la dimensión corpórea tenida en cuenta solamente en su carácter material-físico es lo que Husserl (2005, p.183) denominó Körper. En la lengua alemana hay dos palabras distintas para referirse al cuerpo: Körper y Leib. El Körper se refiere a la dimensión material, física de la corporeidad. Por su parte, el Leib alude a la materialidad del cuerpo en cuanto algo animado, con vida, de hecho, un cuerpo vivido. Entonces resulta que puede decir que el Leib es un Körper animado, pues se manifiesta por medio de la materialidad y, sin embargo, no se reduce a ella, ya que se presenta como algo vivo. Stein prolonga esa distinción de Husserl acentuando ambos matices de la dimensión corpórea.

La constitución material del cuerpo presenta una figura determinada. El Körper no está enmarañado y desordenado en sus distintas partes materiales, pues manifiesta una composición bien demarcada, convirtiendo al hombre en un ejemplo de su especie. De ahí que el cuerpo material esté estructurado en partes como miembros, tronco y cabeza, que determinan una figura corporal concerniente a la especie hombre (Stein, 2002a, p.39). 
Otro matiz del cuerpo material aclarado por Stein es que, así como las demás cosas materiales, también presenta cualidades sensoriales (color, textura, olor, etc.) que entran en conexión causal con los mismos elementos de la naturaleza. También se concibe que el cuerpo material no es algo fijo, pues puede moverse, teniendo en cuenta que está sujeto a cambios causales. Por esta razón, si el cuerpo forma parte del mundo material, él también participa de la legalidad causal de la naturaleza, como, por ejemplo: cuando el cuerpo sufre la acción de la gravedad del mismo modo que cualquier otro objeto físico (Stein, 2005b, p. 789).

En consecuencia, en su aspecto material, el cuerpo humano no tiene ninguna diferencia respecto de los otros objetos y seres. No obstante, se sabe que el cuerpo no es solo algo material, puesto que presenta una peculiaridad que lo distingue de los demás objetos de la naturaleza. De esa manera, hay que preguntar: ¿en qué consiste esa singularidad que diferencia el cuerpo de los otros objetos materiales? ¿Qué hace que él no sea solo material?

La abstracción hecha para investigar el cuerpo material llega así a un límite en que se debe concebir que el cuerpo es más que una simple materia. De esa manera, el cuerpo material no pertenece a un sujeto que tiene un cuerpo, sino que lo vivencia, dado que se presenta como un cuerpo vivido (Leib). De ahí que afirme Stein (2005b, p. 790): "con el vínculo del cuerpo físico vivo para un sujeto o una conciencia individual, se efectúan las peculiaridades que lo caracterizan como cuerpo vivo".

Entonces, el cuerpo vivo es una cosa corpórea material que presenta una íntima conexión con un sujeto consciente, estableciéndose como una materia animada por un sujeto. Husserl (1991, p. 182) nombró esa particularidad en el $\S 53$ de La crisis como la "paradoja de la subjetividad humana", dado que el Yo (Ego) es al mismo tiempo sujeto y objeto para el mundo.

Por consiguiente, al investigar el cuerpo vivido, Stein (2005b, p.790) señaló un aspecto fundamental de su estructura: la impresionabilidad, a saber, la capacidad para tener sensaciones. Concerniente a sus cualidades sensoriales, el cuerpo vivo posee la peculiaridad de ser impresionado. Eso significa que la sensibilidad es una cualidad del cuerpo vivo, que se constituye, de esa manera, como un cuerpo sentiente.

Sin embargo, es posible percibir que el cuerpo vivo posee algo más allá de la facultad de la sensibilidad, ya que también está dotado de movimiento. Mien- 
tras los demás objetos materiales se mueven a causa de algún factor externo, el cuerpo vivo presenta la cualidad de un movimiento propio, eso es, el impulso para su movilidad ocurre desde adentro (Stein, 2002a, p. 53). Antes bien, se constata que el Leib dispone de una configuración interna. Su figura externa se ordena desde dentro, de esa manera se señala una singularidad en el modo de ser de los seres vivos (Stein, 2002a, p. 53).

Aún más, se acentúa que el cuerpo vivo se instituye, de acuerdo con Husserl y Stein, como un órgano de la voluntad. La acción de la voluntad es corporeizada, es decir, solamente puede ser realizada por mediación del cuerpo vivo. Por ejemplo, ante la voluntad de leer un libro, es necesario ir al estante y cogerlo para empezar la lectura. La acción deseada únicamente puede ser llevada a cabo a través de la mediación del cuerpo vivo.

Además de esos aspectos, el cuerpo vivo presenta aún otra singularidad esencial: la capacidad de expresar la vida interior. La vida anímica se caracteriza por seguir la dirección de lo interior al exterior, de modo que ella se explicita por medio del cuerpo vivo. De esa manera, además de posibilitar la expresión de la voluntad, el cuerpo vivo se forma asimismo como un órgano de expresión. La vida se muestra en el cuerpo vivo, imprimiéndole sus peculiaridades. Por eso, es posible aprehender el estado psíquico de una persona por medio de su expresión corporal. Un individuo alegre va a presentarse sonriendo, con el cuerpo afilado y radiante, de manera que empáticamente se capta el estado vital suyo (Stein, 2002a, p. 90; 2005a, p. 240).

Se concluye, así, que el cuerpo vivo, lleno de vitalidad, sensibilidad y movimiento, se presenta como un órgano volitivo y expresivo. Por consiguiente, como apuntó Stein, el Leib es fundamento, expresión e instrumento de vida anímica, puesto que esa vida anímica únicamente puede ocurrir corporalmente, es decir, por intermedio de la estructura corporal, comprendida en cuanto cuerpo-vivo.

Tras el abordaje de la cuestión de la psique y del cuerpo en el análisis fenomenológico de Stein, se está ahora en condiciones de acercarse al problema psique/mente-cuerpo, de la misma manera que se ha anunciado en las ciencias cognitivas. ¿Hay una interacción entre el cuerpo y la psique? ¿Lo corpóreo y lo psíquico son dos substancias distintas? ¿La psique puede ser reducida al cuerpo? Esas son preguntas que se desprenden de tal problema. Por esa razón, tras los análisis que han sido discutidos anteriormente, se trata de exponer cómo se 
establece la cuestión psique/mente-cuerpo a la luz de las reflexiones fenomenológicas hechas por Stein. Hay que señalar que, frente a la complejidad del problema y las limitaciones del presente trabajo, nuestra propuesta no va a ser ofrecer respuestas definitivas, sino reflexionar acerca de esas cuestiones.

\section{EL PROBLEMA PSiQUe/MENTE-CUERPo EN LA FENOMENOLOGÍA DE EDITH STEIN:}

\section{LA UNIDAD CUERPO Y PSIQUE}

Como consecuencia de las limitaciones presentadas en el ámbito de las ciencias de la mente y de la propia psicología, que ofrecen fundamentalmente una mirada fisicalista de la persona, muchos investigadores buscan, por medio de los recursos metodológicos de la fenomenología, considerar la experiencia directa de la persona (subjetiva). Al situar como eje de la investigación al sujeto consciente, la fenomenología se propuso como la base metodológica adecuada para una investigación auténticamente científica de la cuestión.

Con todo, la introducción de la fenomenología en ese debate, en el intento de mantener el ideario científico, ha ocurrido por medio del proyecto de naturalización, que presenta serios equívocos. Naturalizar la fenomenología parece quitarle su carácter genuinamente trascendental, algo propio de ella. Por tanto, si el proyecto de naturalización demostró que no es la vía adecuada para la inserción de la fenomenología en las discusiones sobre la cuestión mente-cuerpo, es necesario subrayar cómo es posible esa interlocución. ¿Cómo el carácter trascendental de la fenomenología puede ayudar en la investigación científica del problema psique/mente-cuerpo? Aunque parezca contradictorio, la interlocución de la fenomenología trascendental con el sujeto empírico ocurre, primeramente, por una vía negativa (Sidoncha, 2011, p. 60). A causa del carácter encarnado del "Yo-empírico", se hace, ante todo, una suspensión de su inscripción en el mundo natural. Como destacó Husserl (2006, p. 73) y Stein (2005a, p. 224), a fin de alcanzar la esfera de las vivencias puras es necesario interrumpir cualquier resquicio de la orientación natural. Así, se promueve una abstracción del "Yo-empírico", buscando alcanzar el sujeto trascendental.

Por eso, puede cuestionarse si esa abstracción implica una exclusión del "Yo-empírico". A pesar de las acusaciones de idealismo trascendental de los propios discípulos del Fenomenólogo, Husserl (2005, p. 133) y Stein (2005a, p. 236) mostraron que, fuera de excluir, las investigaciones fenomenológicas bus- 
can exponer las condiciones de posibilidad de lo que ha sido suspendido. Al comprender el sujeto trascendental como condición de posibilidad del "Yoempírico", es inevitable que este sea en primer momento suspendido para luego ser recuperado por aquel, ahora de manera originaria.

Más aún, al explicitar el carácter constitutivo de la conciencia, Husserl (2005, p. 133) y Stein (2005a, p. 236) trataron de analizar el proceso de constitución. Todo lo que ha sido suspendido por medio de las herramientas del método fenomenológico (Epoché, reducción) se restauró de modo originario a través del proceso constitutivo. Ante lo expuesto, se concluye que el análisis constitutivo se establece como el camino más adecuado con el fin de la investigación fenomenológica del sujeto empírico y, en consecuencia, de la cuestión psique/mente-cuerpo. Como se explicitó, al seguir las reflexiones de su maestro, Stein (2005a, p. 236) emprendió un provechoso análisis de la psique y del cuerpo.

En su investigación, la filósofa (2005a, p. 224; 2005b, p. 778) analizó, desde el "Yo-puro", cómo se constituye el "Yo-empírico" (psicológico). Sin embargo, ¿en qué consiste ese "Yo-empírico" o real? ¿Él se forma por dos sustancias distintas (cuerpo y mente) como afirmó Descartes? ¿O, por otro lado, es solamente algo físico tal como lo han propuesto las teorías fisicalistas? Con objeto de contestar a esas cuestiones, se recurre a las investigaciones fenomenológicas hechas por Stein acerca de la psique y del cuerpo.

Según Stein (2005a, p. 236; 2005b, p. 787) y Husserl (2005, p. 125) evidenciaron, la realidad presenta dos matices distintos: la naturaleza material y la naturaleza animal (psíquica); en otras palabras, hay una realidad material y otra psíquica. No obstante, se acepta una concepción unitaria de la realidad, la que es comprendida como la totalidad de las cosas reales. De esa totalidad se destaca el aspecto únicamente material y también lo psíquico, por ejemplo, el ámbito de las cosas animadas.

Así, el cuerpo presenta un aspecto distintivo, pues comparte dos características distintas. Al mismo tiempo que es algo material, exhibe un carácter vivo, pues es una cosa animada. Corroborando lo que había escrito Stein (2002a, p. 39; 2005a, p. 293; 2005b, p. 788), el cuerpo es simultáneamente una simple materia (Körper) y organismo vivo (Leib). De ahí que el cuerpo vivo, animado por la psique, muestre la peculiaridad de compartir tanto la naturaleza material como la psíquica. Es relevante resaltar que la distinción que hizo Stein entre los 
diferentes aspectos de la corporeidad, Körper y Leib, no establece un dualismo en la dimensión corpórea como se podría equivocadamente pensar. Körper y Leib no son cuerpos distintos, sino distintas cualidades de un mismo cuerpo.

El cuerpo vivo (Leib) es simultáneamente objeto y sujeto para el mundo, pues es una materia animada, a causa de su íntimo vínculo con un sujeto. Stein (2005b, p. 815) afirmó que el Leib se pone como el origen o el marco cero de orientación del sujeto (Nullpunkt). Así, no se percibe el cuerpo de la misma manera que las demás cosas materiales, ya que él es el punto en que sucede la percepción y la autopercepción.

Al tomar un objeto material se concluye que él es distinto del cuerpo, ya que está fuera del sujeto, a saber, es algo trascendente. Ahora bien, el sentido del tacto tiene una importancia fundamental a ese respecto, puesto que a través de él es posible delimitar los límites del cuerpo. Por ejemplo, en el momento en que un niño explora un ambiente, cuando tantea los objetos, todas esas operaciones le ayudan a establecer las fronteras de su Yo, en cuanto lo que es tanteado es percibido como distinto de él. De ahí que el cuerpo es el punto desde el cual el sujeto se orienta.

El sujeto no es una psique que se ubica en un cuerpo, sino un individuo corpóreo-psíquico. El cuerpo vivo solamente se constituye como una materia animada a causa de su íntima conexión con la psique. En contrapartida, Stein (2005b, p. 815) destacó que la psique solamente se manifiesta a través de su relación con lo material, lo que constituye así el cuerpo vivo. Entonces, se entiende la imposibilidad para que los fenómenos psíquicos ocurran aisladamente, fuera del dominio del Leib.

De esa manera, en consonancia con Husserl, Stein concibe el Leib como una unidad entre materia y psique. Como destacaron Bello \& Manganaro (2012, p. 13), ese enlace puede ser entendido, como sugirió Stein, a modo de una "unidad-dual". Lo que quiere decir que, aunque el cuerpo y la psique sean dos estructuras distintas y que no se mezclan, se entrelazan y ambos solamente se constituyen desde una unidad.

La comprensión del vínculo cuerpo-psique como una unidad-dual, al mismo tiempo que busca resaltar el dual aspecto del problema, eso es, el cuerpo y la psique, entiende que ambos interactúan de cara a formar una unidad. Así, se comprende que el cuerpo y la psique no se manifiesten aisladamente, pues se constituyen solamente en el ámbito de esa unidad. De esa manera, a través de 
la noción de Leib, se entiende que la unidad-dual posee como fundamento el cuerpo vivo, pues es en él donde se establece la unión corpóreo-psíquica. (Bello \& Manganaro, 2012, p. 13).

A través de esa mirada, se busca conservar ambos elementos del problema, considerando la peculiaridad de cada uno y, de esa manera, evitar una concepción reduccionista. Sin embargo, se puede objetar que una unidad-dual consiste en una posición dualista, al considerar dos dominios distintos, cuerpo y psique. Con todo, aunque evidencie un aspecto dual, esa mirada no implica un dualismo, ya que en el análisis fenomenológico de las experiencias en Stein (2002a, p. 126 ; 2005a, p. 293; 2005b, p. 815) es posible identificar que esa dualidad únicamente se manifiesta por medio de una unidad. Incluso se destaca que ambos elementos (cuerpo y psique) no se presentan como cosas, como res, o sea, una sustancia, sino como distintos aspectos esenciales de una misma realidad.

Además, se puede resaltar, respecto a esa perspectiva, que, al concebir una unidad, se desencadena una faceta monista. Con todo, la unidad-dual tampoco señala que la realidad esté formada por solamente un matiz, como el monismo entiende. De acuerdo con lo destacado, la posición monista subraya la existencia de un solo dominio, tanto físico como mental, lo que depende de la modalidad asumida, el fisicalismo o el idealismo respectivamente. Se nota que, en esa postura, uno de los elementos del problema psique/mente-cuerpo es desdeñado; en el fisicalismo, el aspecto mental es devaluado mientras que en el idealismo se desvalúa el elemento físico.

No obstante, a través de la noción de una unidad-dual, sugerido en la acepción de Leib en Stein (2002a, p. 126; 2005b, p. 815), se comprende que esa unidad no indica la consideración de que la realidad esté formada únicamente por un elemento. Ante eso, se destaca que en esa concepción, aunque se presente una unidad, se busca considerar los distintos aspectos que la forman (cuerpo y psique). Así, no se permite entender la perspectiva de una unidaddual como un monismo, sea este fisicalista, sea idealista. En consecuencia, se busca valorar ambos elementos de la cuestión, en el sentido de una armonía de esos distintos aspectos. Aunque sean distintos, esos dominios, cuerpo y psique, se armonizan en una unidad que los constituyen (Bello, 2015, p. 51).

Esa comprensión posibilita establecer una prioridad de lo psíquico o, mejor, del Leib, puesto que la psique no se manifiesta además del cuerpo vivo, y este 
solamente se constituye como algo vivo a causa de su vínculo con un sujeto psíquico. De esa manera, como apuntan Bello \& Mangarano (2012, p. 23), es necesario establecer una distinción entre lo que es la fuente, o sea, lo originario, y lo que es la base, que da soporte. Las investigaciones de la neurociencia, por ejemplo, afirman que el cerebro es la fuente originaria de las experiencias $y$, al afirmar que determinado fenómeno psíquico se identifica con una región del cerebro, se entiende que esa ubicación cerebral es lo que da origen a la experiencia psíquica. La hipótesis básica de las ciencias del cerebro es que el sustrato material se reconoce como la fuente originaria de la vivencia psíquica.

Al mismo tiempo, es esencial cuestionar ese enfoque, indagando si esa base material es, en realidad, la fuente de la vida anímica. Ante las limitaciones de las ciencias de la mente, se comprueba que la perspectiva fisicalista no engloba la experiencia subjetiva (primera persona), ya que el lenguaje impersonal de las neurociencias no dice nada respecto a las cualidades subjetivas de la experiencia. Se percibe, así, que hay elementos que van más allá del aspecto material.

Dicho de otra manera, resulta que el sustrato material no es la fuente originaria de las experiencias psíquicas, sino su base. Es evidente que, como mostró la teoría de la superveniencia, los fenómenos mentales son soportados por una estructura material, puesto que sería inconcebible la realización de estados mentales sin el cerebro. Sin embargo, el sustrato material no constituye su fuente, sino la base de las vivencias.

Desde esos apuntes, se constata que la fuente originaria de la vida anímica es la psique, pues el cuerpo es su sustrato, su sustentación, su base. El cerebro participa de la vida anímica de manera secundaria, ya que su funcionamiento ocurre debido al hecho de constituirse como parte de un cuerpo animado por un sujeto psíquico. Lejos de desvalorar, la fenomenología busca resaltar el carácter originario de lo psíquico, no obstante, sin ignorar su sustrato material. Desde la noción de unidad-dual se considera tanto el aspecto material como el psíquico, señalando el carácter de ambos. Mientras este constituye la fuente de la vida anímica, aquel se establece como la base (Bello \& Mangarano, 2012, p. 23).

La comprensión del vínculo cuerpo-psique como una unidad-dual aceptada explícitamente por la fenomenología de Stein cuestiona y pone en jaque el modelo reduccionista de lo mental. Según el fisicalismo reduccionista, toda ocurrencia psíquica se reduce al sustrato físico, el cual dice respecto a su base ex- 
plicativa u ontológica que depende del tipo de reduccionismo. De esa manera, la tesis principal del modelo reduccionista es que toda la vida psíquica se agota en las estructuras biológicas del cuerpo material, a saber, el Körper.

Por esta razón, en contra de la preponderancia del material defendido por las teorías reduccionistas, la fenomenología sostiene una inequívoca prioridad del Leib, de la unidad-dual corpóreo-psíquica. Desde esa concepción, se está en situación de superar el modelo reduccionista, el que comprende el cuerpo como algo únicamente material, promoviendo una devaluación de la psique. Con todo, las investigaciones fenomenológicas evidencian que el cuerpo no es una simple materia, visto que es animado a causa de su enlace con la psique (Sidoncha, 2011, p. 120).

Por estas razones resulta viable constatar los errores de los análisis hechos por la psicología y las ciencias cognitivas acerca de la mente. Ante la falta de aclaración respecto a lo psíquico, esas áreas de investigación lo conciben equívocamente, lo que hace percibir la necesidad de una investigación rigurosa de la psique (Stein, 2005a, p. 217). De manera general, las investigaciones psicológicas y cognitivas entienden lo psíquico desde una concepción fisicalista, reduccionista y cuantitativa, expresando sus conclusiones por medio de un lenguaje impersonal.

Sin embargo, la cuestión es si ese tipo de investigación logra abarcar eficazmente la totalidad del ser humano. Así, hay que subrayar que la psicología y las ciencias cognitivas piensan al ser humano como algo únicamente material y a lo psíquico con matices físicos. Según los análisis de Husserl (2005, p. 125) y Stein (2002a, p. 34; 2005a, p. 236; 2005b, p. 787), el ser humano comparte con las demás cosas de la naturaleza una dimensión material, es decir, el cuerpo, entendido como Körper. Sin embargo, se distingue de los demás objetos, pues el cuerpo no es algo solamente material. El Körper está animado por una dimensión psíquica, constituyendo así una unidad-dual corpóreo-psíquica que se instaura desde el cuerpo vivo (Leib).

Por ende, se comprende que una psicología verdaderamente científica necesite hacer una rigurosa investigación del Leib, entendiendo que la psique se manifiesta solamente por medio de la unidad-dual corpóreo-psíquica. Con esos fundamentos se infiere que todas estas distinciones pueden contribuir notablemente a una adecuada aclaración del problema psique/mente-cuerpo. La noción de Leib, comprendido este como una unidad-dual, aparenta constituirse como 
una propuesta pertinente para la discusión de esa cuestión. No obstante, lejos de haber agotado aquí el tema, se requeriría una investigación posterior con el objetivo de alcanzar una comprensión rigurosa y fundamentada del problema psique/mente-cuerpo.

\section{REFERENCIAS}

Araújo, S. d. (2007), "Wilhelm Wundt e o estudo da experiência imediata", en: A. M. Jacó-Vilela, A. A. Ferreira, \& F. T. Portugal, História da Psicologia: rumos e percursos, pp. 93-104. Rio de Janeiro: Nau Editora.

Bello, A. A. (2015), Pessoa e Comunidade, trads I. J. Garcia, \& M. Mahfoud. Belo Horizonte: Artesão.

- (2012). "Status quaestionis". en A. A. Bello, \& P. Manganaro 2102, pp. 1338.

- \& Manganaro, P. (2012), E La Coscienza? fenomenología Psico-Patologia Neuroscienze. Bari: Edizione Giuseppe Laterza.

Depraz, N. (1999), "When Transcendental Genesis Encounters the Naturalization Project". en J. Petitot, F. J. Varela, B. Pachoud, \& R. Jean-Michel, Naturalizing Phenomenology, pp. 464-489. Stanford: Stanford University Press.

Goto, T. A. (2015), Introdução à Psicologia Fenomenológica - A nova Psicologia de Edmund Husserl. São Paulo: Paulus.

Hussert, E. (1965), Filosofia como ciência de rigor, trad A. Beau. Coimbra: Atlântida.

HUSSERL, E. (2005), Ideas relativas a una fenomenología pura y una Filosofia Fenomenológica. Libro segundo: Investigaciones fenomenológicas sobre la constitución, trad. de A. Z. Quijano. México: Fondo de Cultura Económica.

- (2006), Ideias para uma fenomenología pura e para uma Filosofia Fenomenológica. Aparecida: Ideias \& Letras.

- (1991). La crisis de las ciencias europeas y la fenomenología trascendental, .trad. de J. Muñoz, \& S. Mas. Barcelona: Editorial Crítica.

MAnganaro, P. (2012), "Materia (I) Mente? Le «ragioni» del corpo e l'empatia", en P. Manganaro, \& A. A. Bello 2012, pp. 241-377. Roma: Edizioni Giuseppe Laterza.

SidonchA, U. M. (2011), Do Empírico ao Transcendental: a consciência e o problema mente/corpo entre o materialismo reducionista e a fenomenologia de Husserl. Beira Interior: Fundação Para a ciência e a Tecnologia.

- (2008). "O Debate - Possível - Entre Materialismo Reducionista e fenomenología", en Estudos e Pesquisas em Psicologia , 8, pp. 384 - 401.

SimANKE, R. T. (2012). "Um ponto cego no programa de naturalização da fenomenologia", en S. d. (Org.), História e Filosofia da Psicologia: perspectivas contemporâneas, pp. 321-337. Juiz de Fora: Editoria UFJF. 
Stein, E. (2005a), Causalidad psíquica. en E. Stein, Obras Completas II: Escritos Filosóficos - Etapa Fenomenológica, trads. C. R. Garrido, \& J. L. Bono, pp. 217-342. Burgos: Editorial Monte Carmelo.

- (2007), "El castillo Interior", en E. Stein, Obras completas III: Escritos filosóficos - Etapa del pensamiento cristiano, pp. 1113-1136. Burgos: Editorial Monte Carmelo.

- (2005b), Introducición a la Filosofía. en E. Stein, Obras completas II: Escritos Filosóficos - Etapa Fenomenológica, trads. C. R. Garrido, \& J. L. Bono, , pp. 657-913. Burgos: Editorial Monte Carmelo.

- (2002a), La Estructura de la Persona Humana. Madrid: Biblioteca de Autores Cristianos.

- (2002b), Vida de una familia judía. en E. Stein, Obras Completas I: Escritos Autobiográficos y Cartas, trads. J. G. Rojo, E. G. Rojo, J. Sancho, C. RuizGarrido, \& J. Urkiza. Burgos: Editorial Monte Carmelo.

ZAHAVI, D. (2010), "Naturalized Phenomenology", en S. Gallagher \& D. Schmicking, Handbook of Phenomenology and Cognitive Science, pp. 3-19. New York: Springer. 